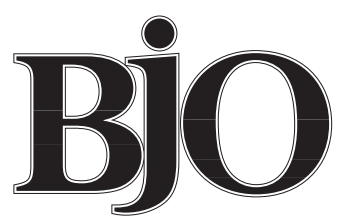

British Journal of Ophthalmology

Cataract extraction is of undisputed efficacy in terms of improvements in both vision and quality of life. However, the benefits (and risks) of cataract surgery in patients with concomitant ocular diseases, such as age related macular degeneration (ARMD), are uncertain. With rapidly ageing populations and greater life expectancy, the number of patients with both cataract and ARMD will inevitably increase. How do we manage these patients? Is cataract surgery justified? Are there some who will benefit more than others? Conversely, does cataract surgery aggravate ARMD in other patients? Reliable data are lacking, but these issues are clearly important.

The paper by Armbrecht and colleagues in this issue of the $B F O$ ( $\mathrm{p}$ 1343) therefore represents an important and timely contribution to the literature. In this prospectively designed study of 187 patients, 90 with ARMD who underwent cataract surgery and two control groups (one with ARMD without cataract surgery and another without ARMD but with cataract surgery), the authors found significant improvements in both visual function and quality of life in patients with ARMD who had cataract surgery. Specifically, patients with mild and moderate ARMD benefited most from cataract surgery, especially if cataract was of moderate severity. Thus, this study provides data that suggest cataract surgery should be offered to selected patients with moderate cataract and mild/moderate ARMD, challenging the traditional view that most patients with ARMD are unlikely to benefit (and may even be harmed) from the procedure.

In a sense, the relation between cataract surgery and ARMD has been inconsistent. While some epidemiological studies have suggested an increased incidence and progression of ARMD among people who had cataract surgery, others have not found an association. In the Beaver Dam Eye study, Klein and colleagues found that eyes that had undergone cataract surgery were more likely to have a 5 year progression of age related maculopathy (OR $2.7 ; 95 \% \mathrm{CI}, 1.7-4.4)$ and to develop signs of late age related maculopathy (OR 2.8; 95\% CI, 1.0-7.6) than were eyes that were phakic, with the association significant despite controlling for other ARMD risk factors. ${ }^{1}$ However, in other large epidemiological studies, the Blue Mountains Eye Study and the Rotterdam Eye Study, no cross sectional association between cataract surgery and ARMD was observed. ${ }^{2} 3$
Fears that cataract surgery may be associated with higher risk of ARMD progression also arose from data based on clinical studies by Pollack et $a l,{ }^{4-6}$ and from postmortem pathological studies by Van der Schaft et $a l^{7}$ However, as pointed out by Armbrecht and colleagues, there are important limitations in these studies that suggest the results were inconclusive, and if anything, warrant further research. ${ }^{8}$ One such study by Shuttleworth and colleagues showed a clear benefit from cataract surgery in the majority of patients (more than two thirds) with ARMD. ${ }^{9}$ Nevertheless, the study was retrospective in nature (and subjected to recall and other bias) and the prevalence of ARMD in that study population was lower than expected (suggesting selective exclusion of patients with both ARMD and cataract). Armbrecht and colleagues' contribution in this issue of the $B \mathcal{F O}$ is important in two respects. Firstly, the prospective design, semistandardised grading of both cataract and ARMD, and objective assessment of visual function and quality of life represents additional improvements over Shuttleworth et al's study. Secondly, the study identified subgroups of patients who are more likely to benefit from cataract surgery; not unexpectedly, these were patients with mild or moderate ARMD and with moderate (and presumably more severe) cataract. However, caution is advised for several reasons. The study is limited by a short follow up period ( 5 months), inadequate power to detect progression of ARMD (particularly in the subgroup of patients with severe ARMD, $n=8$ ), possible inconsistencies in ARMD grading between the two study centres (direct ophthalmoscopy in Oxford, and photographic grading in Edinburgh), and selection bias inherent in any non-randomised design.

Where then do we go from here? There are several options. Firstly, an obvious "next step" is to consider a randomised clinical trial. This is not feasible as there will be ethical issues (for example, patients with mild cataract and moderate ARMD) and problems related to randomisation, masking, and objective assessment of benefits versus risks. If a clinical trial is not feasible, further prospective studies will provide much needed supporting evidence. These studies should preferably be multicentred and involve well defined prospective cohorts. They should be of sufficient power to detect significant difference in benefits and risks in different subgroups (combinations of mild/moderate/severe cataract and ARMD). These studies should have objective and standardised documentation of 
both cataract and ARMD severity, as well as preoperative and postoperative assessment of visual and quality of life outcomes. Some form of masking of the observers is critical. Finally, we have to adhere to the basic ethical principle of medicine: first do no harm, and remember that cataract surgery may, in some cases, subject patients to higher risk of blindness as a result of complications, such as progression of ARMD. Thus, certain high risk groups should be omitted from such a study.

Armbrecht et al's article has clearly started the ball rolling again on this very important problem. In the context of the data from this paper it appears that cataract surgery in patients with mild ARMD and moderate to severe cataract may be beneficial, but we should probably still be conservative in our management of patients with more severe ARMD and with mild cataracts. We await eagerly further studies to provide a consistent, reliable, and accurate answer to a question posed commonly by our patients with cataract and ARMD, "what are my chances of seeing better after a cataract operation?"

TIEN YIN WONG

Singapore National Eye Centre, Singapore; Department of Ophthalmology and Visual Sciences, University of Wisconsin, Madison, WI, USA; and Department of Epidemiology, Johns Hopkins University, Baltimore, MD, USA
Correspondence to: Department of Ophthalmology and Visual Sciences, University of Wisconsin-Madison, 610 North Walnut Street, 460 WARF, Madison, WI 53705-2397, USA

wong@epi.ophth.wisc.edu

1 Klein R, Klein BE, Jensen SC, et al. The relationship of ocular factors to the incidence and progression of age-related maculopathy. Arch Ophthalmol 1998;116:506-13.

2 Wang JJ, Mitchell PG, Cumming RG, et al. Cataract and age-related maculopathy: the Blue Mountains Eye Study. Ophthalmic Epidemiol 1999;6:317-26.

3 Vingerling JR, Klaver CCW, Hofman A, et al. Cataract extraction and age related macular degeneration: the Rotterdam Study [abstract]. Invest $O p h$ thalmol Vis Sci 1997;38:S472.

4 Pollack A, Bukelman A, Zalish M, et al. The course of age-related macular degeneration following bilateral cataract surgery. Ophthalmic Surg Lasers 1998;29:286-94.

5 Pollack A, Marcovich A, Bukelman A, et al. Development of exudative age-related macular degeneration after cataract surgery. Eye 1997;11:52330.

6 Pollack A, Marcovich A, Bukelman A, et al. Age-related macular degeneration after extracapsular cataract extraction with intraocular lens implantation. Ophthalmology 1996;103:1546-54.

7 Van der Schaft TL, Mooy CM, de Bruijn WC, et al. Increased prevalence of disciform macular degeneration after cataract extraction with implantation of an intraocular lens. Br f Ophthalmol 1994;78:441-5.

8 Armbrecht AM, Findlay C, Aspinall P, et al. Do patients with age related maculopathy and cataract benefit from cataract surgery? Br $\mathcal{F}$ Ophthalmol 1999;83:252.

9 Shuttleworth GN, Luhishi EA, Harrad RA. Do patients with age related maculopathy and cataract benefit from cataract surgery? $\mathrm{Br} \mathcal{F}$ Ophthalmol 1998;82:611-6.

\section{Bleb related ocular infection: a feature of the HELP syndrome}

Glaucoma is a progressive optic neuropathy which represents the final common pathway of a number of different disorders which affect the eye. Most, but not all, of these are associated with elevated intraocular pressure (IOP), which is the most important known risk factor for optic nerve damage, but is still only a risk factor and not the disease itself. The progressive loss of retinal ganglion cells and their axons leads to a characteristic clinical pattern of optic nerve head and corresponding visual field damage. Non-IOP independent mechanisms of glaucomatous damage are not confined to "normal tension" glaucoma, but can be operative in any patient, although they predominate when glaucomatous damage occurs at the lower end of the pressure spectrum. The goal of glaucoma filtering surgery is to reduce or eliminate the pressure dependent component of the disease process.

Filtration surgery to lower IOP has been in use for over a century. Limbal trephination, described by Elliot in $1909,{ }^{1}$ became the most popular operation until the 1940 s, when it fell out of favour because the very thin conjunctival bleb predisposed to late endophthalmitis. Thermal cautery combined with peripheral iridectomy, ${ }^{2}$ and posterior lip sclerectomy, ${ }^{3}$ became the most widely used operations until guarded filtration procedures were developed. All of these full thickness procedures, however, were complicated by frequent flat anterior chambers, ciliochoroidal detachments, occasional choroidal haemorrhages, and a high incidence of subsequent cataract formation. Late endophthalmitis was common.

Improvements in microsurgical instrumentation and fewer postoperative complications led to the rapid acceptance of guarded filtration techniques following the introduction of trabeculectomy by Cairns in $1968 .{ }^{4}$ Although the incidence of late endophthalmitis was significantly reduced, the IOP was not lowered to as great an extent and certain subsets of patients, such as those with neovascular glaucoma, uveitis, and previous surgery had high rates of failure. The introduction of 5-fluorouracil (5-FU) and mitomycin C (MMC) markedly improved the surgical success rates not only in these eyes, but also in routine filtration surgery, so much so that most glaucoma surgeons use antifibrosis agents for virtually all cases, in an attempt to provide the pressure lowering effect of full thickness surgery with the safety of trabeculectomy. Combined with postoperative laser suture lysis, allowing tighter scleral flap closure, and earlier postoperative manipulation to encourage bleb formation, complications such as flat anterior chamber have been markedly reduced. However, the thin, avascular, cavitated blebs produced, particularly with MMC, have led to a resurgence of chronic hypotony, bleb leaks, bleb infections, and endophthalmitis. Many of these patients also complain of ocular pain or discomfort related to the presence of a large or elevated bleb, a condition known as bleb dysethesia. We have termed the postoperative constellation of complications associated with antifibrosis agents the HELP syndrome (hypotony, endophthalmitis, leak, pain).

In this issue of the BFO ( $p$ 1349), Lehmann and colleagues have assessed risk factors for the development of late endophthalmitis following glaucoma filtering surgery. In a well designed, retrospective, case-controlled series, they report that bleb infection, diabetes mellitus, and use of antifibrosis agents increase the risk of developing late endophthalmitis. Patients receiving antifibrosis agents tended to develop infection considerably earlier than those individuals who did not receive them. They attribute this increased risk of infection to the altered bleb morphology (thin walls, denuded epithelium, abnormal stroma, leakage, avascularity) characteristic of 5-FU and MMC filter- 
ing blebs. Endophthalmitis led to a significant loss of vision and bleb function in a large proportion of eyes.

This article is important for several reasons. Firstly, it supports the findings of others. Since our initial report of late bleb related ocular infection following 5-FU trabeculectomy ${ }^{5}$ and the coining of the term "blebitis", 6 we have treated many patients with late bleb related complications and infections. These reports have included children ${ }^{7}$ recurrence in previously infected eyes, ${ }^{8}$ and institution-wide surveillance documenting 49 episodes (42 patients) over a 10 year period, ${ }^{9}$ with bleb leakage being a common predisposing condition to both infection and associated with antifibrosis use. ${ }^{10}{ }^{11}$ Secondly, the article by Lehmann and colleagues documents that this problem is worldwide in scope. Late bleb related ocular infection involves all ethnic and racial groups, although the infecting organisms may vary geographically. Thirdly, bleb related infection can be a serious cause of permanent vision loss and may interfere with bleb function. An association with diabetes may reflect the greater predisposition of such patients to infection in general, and might give us pause to consider in which subsets of patients it might be advisable to exercise greater caution in deciding whether or not to use MMC. Finally, the article confers renewed attention on this important aspect of the HELP syndrome and on our need to rethink the use of these potent compounds.

The morbidity associated with HELP syndrome should cause us to reflect on the widespread, often indiscriminate, use of antifibrosis agents. Although the rate of progressive glaucoma damage is lower the lower the postoperative IOP, not every patient requires an IOP in the single digits or low teens to prevent further significant injury. Most patients would be able to retain useful vision for the duration of their lives with somewhat higher pressures and we should tailor our therapeutic intervention to the particular needs of the individual patient, rather than reach, often reflexively and without thought, for the application of MMC. The literature comparing the efficacy of adjunctive 5-FU with MMC for uncomplicated, previously unoperated eyes is surprisingly scant, and a recent report by Singh and colleagues suggests that the pressure lowering effect is similar for these two drugs in this group of patients. ${ }^{12}$ Some might argue that MMC be reserved primarily for eyes with extremely advanced damage, reoperation, or complicated or combination surgeries. Each individual surgeon needs to reassess his or her use of these medications based upon personal experience, patient population, and spectrum of patient disease severity, and make a thoughtful decision regarding the appropriate, judicious use of these medications.

Looking to the future, is it possible to achieve our IOP reduction goals without the severe alteration of bleb morphology present following the use of 5-FU or MMC? The
Moorfields group has been at the forefront of the search for safer, more effective, and more physiological means of wound healing modification. An excellent review of the topic of potential avenues for immune modification in wound healing has recently been published. ${ }^{13}$

Transforming growth factor $\beta$ (TGF $\beta$ ) is a potent stimulator of human Tenon's capsule fibroblast activity, suggesting its stimulatory role in the conjunctival scarring response. ${ }^{14-16}$ The use of antihuman TGF $\beta$ antibody in reducing conjunctival wound healing is one such innovative avenue. ${ }^{17}$ We look forward to seeing the results of human clinical trials currently under way.

Supported by the Donald Engel Research Fund of the New York Glaucoma Research Institute, New York, USA.

\section{JEFFREY M LIEBMANN} ROBERT RITCH

Departments of Ophthalmology, The New York Eye and Ear Infirmary, New York, NY and New York Medical College, Valhalla, NY, USA

Correspondence to: Dr Robert Ritch, Glaucoma Service, The New York Eye and Ear Infirmary, 310 East 14th Street, New York, NY 10003, USA ritch@inx.net

1 Elliot RH. A preliminary note on a new operative procedure for the establishment of a filtering cicatrix in the management of glaucoma. The Ophthalmoscope 1909;7:804-6.

2 Scheie HG. Retraction of scleral wound edges: a fistulizing procedure for glaucoma. Am f Ophthalmol 1958;45:220-9.

3 Iliff CE, Haas JS. Posterior lip sclerectomy. Am f Ophthalmol 1962;54:688. 4 Cairns JE. Trabeculectomy-preliminary report of a new method. Am $\mathcal{f}$ Ophthalmol 1968;66:673-9.

5 Wolner B, Liebmann JM, Sassani JW, et al. Late bleb-related endophthalmitis after trabeculectomy with adjunctive 5-fluorouracil. Ophthalmology

6 Brown RH, Yang LH, Walker SD, et al. Treatment of bleb infection after glaucoma surgery. Arch Ophthalmol 1994;112:57-61.

7 Waheed S, Ritterband DC, Greenfield DS, et al. Bleb-related ocular infection in children after trabeculectomy with mitomycin C. Ophthalmology 1997;104:2117-20

8 Waheed S, Liebmann JM, Greenfield DS, et al. Recurrent bleb infections. $\mathrm{Br}$ f Ophthalmol 1998;82:926-9.

9 Waheed S, Ritterband DC, Greenfield DS, et al. New patterns of infecting organisms in late bleb-related endophthalmitis: a ten year review. Eye 1998; 12:910-15.

10 Soltau JB, Rothman RF, Budenz DL, et al. Risk factors for glaucoma filtering bleb infections. Arch Ophthalmol 2000;118:338-42.

11 Greenfield DS, Liebmann JM, Jee J, et al. Late-onset bleb leaks after glaucoma filtering surgery. Arch Ophthalmol 1998;116:443-7.

12 Singh K, Mehta K, Shaikh N, et al. Trabeculectomy with intraoperative mitomycin $C$ versus 5-fluorouracil: prospective randomized clinical trial. Ophthalmology (in press)

13 Chang L, Crowston JG, Cordeiro MF, et al. The role of the immune system in conjunctival wound healing after glaucoma surgery. Surv Ophthalmol in conjunctival

14 Cunliffe IA, Rees RC, Rennie IG. The effect of TGF-beta 1 and TGF-beta 2 on the proliferation of human Tenon's capsule fibroblasts in tissue culture. Acta Ophthalmol 1996;74:31-5.

5 Khaw PT, Occleston NL, Schultz G, et al. Activation and suppression of fibroblast function. Eye 1994;8:188-95.

16 Cordeiro MF, Bhattacharya SS, Schultz GS, et al. TGF-beta1, -beta2, and beta3 in vitro: biphasic effect on Tenon's fibroblast contract, proliferation, and migration. Invest Ophthalmol Vis Sci 2000;41:756-63.

17 Cordeiro MF, Gay JA, Khaw PT. Human anti-transforming growth factor- $\beta 2$ antibody: a new glaucoma anti-scarring agent. Invest Ophthalmol Vis Sci 1999;40:2225-34.

\section{New techniques in glaucoma surgery}

Recently, new techniques have been introduced for performing glaucoma surgery without opening into the anterior chamber, thus avoiding the complications which are commonly associated with penetrating glaucoma surgery. These methods involve exposure of the canal of Schlemm under a deep scleral flap without actually entering the anterior chamber and sometimes insertion of a collagen sponge under the scleral flap. ${ }^{1-4}$
Essentially these methods stem from the seminal work of Grant who demonstrated that in normal enucleated human eyes $75 \%$ of the resistance to aqueous outflow was located in the trabecular meshwork. ${ }^{5}$ Following Grant's work, various attempts were made to redesign glaucoma surgery to remove resistance at the trabecular meshwork thus lowering intraocular pressure. It is instructive now to reappraise these attempts. 
Redmond Smith ${ }^{6}$ introduced the concept of trabeculotomy as surgery for open angle glaucoma and this was further developed by Harms and Dannheim. ${ }^{7}$ Soon after Grant's work Dvorak-Theobald and Kirk $^{8}$ pointed out that some cases of open angle glaucoma were due to obstruction of the scleral collectors and Krasnov introduced sinusotomy for use in glaucoma when trabecular function appeared adequate. ${ }^{9}$ Sinusotomy and trabeculotomy were even combined to produced a filtering bleb with an intact anterior chamber. ${ }^{10}$

In 1968 John Cairns introduced trabeculectomy having noted the lack of acceptance of trabeculotomy. ${ }^{11}$ Essentially this functions as a guarded full thickness sclerectomy, although Cairns originally postulated that removal of trabecular meshwork would allow free flow of fluid into the open lumen of the canal of Schlemm bypassing trabecular resistance. There is now little doubt that trabeculectomy has supplanted all other forms of glaucoma surgery.

This early experience is probably relevant to the recent innovations. The lack of randomised, prospective, casecontrol trials with adequate follow up for the new procedures has recently been criticised. ${ }^{12}{ }^{13}$ This was remedied in the $B \mathcal{B O}$ last year for cases with a collagen implant $^{14}$ although various criticisms have remained, including the risk of perforation of the trabecular meshwork and the frequent need for YAG laser trabecular puncture postoperatively. The criteria of success include a pressure of less than $21 \mathrm{~mm} \mathrm{Hg}$, hardly acceptable to most current workers in glaucoma for advanced cases and topical medication is often needed to achieve this.

In the light of the complexity of the procedures proposed, the lack of careful study of the trabecular meshwork is notable although some early studies have been

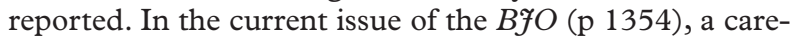
ful study of the trabecular meshwork in glaucoma is presented stressing the variability of the tissue and that the anterior meshwork is extremely thin in older patients. This makes perforation of the mesh at operation likely even for experienced surgeons.

In view of these findings and remembering our previous experience with surgery of the trabecular mesh, it seems wise to withhold judgment on deep sclerectomy and viscocanalostomy until more extensive clinical appraisal is available.

ANNE M V BROOKS W E GILLIES

The Royal Victorian Eye and Ear Hospital, Melbourne, Australia abrooks@medeserv.com.au

1 Stegmann R, Pienaar A, Miller D. Viscocanalostomy for open-angle glaucoma in black African patients. F Cataract Refract Surg 1999;25:31622 .

2 Mermoud A, Schnyder CC, Sickenberg M, et al. Comparison of deep sclerectomy with collagen implant and trabeculectomy in open angle glaucoma. F Cataract Refract Surg 1999;25:323-31

3 Welsh MH, DeLange J, Wasserman P, et al. The "deroofing" of Schlemm's canal in patients with open-angle glaucoma through placement of a collagen drainage device. Ophthalmic Surg Lasers 1998;29:216-26.

4 Carassa RG, Bettin P, Fiori M, et al. Viscocanalostomy: a pilot study. Eur 7 Ophthalmol 1998;8:57-61.

5 Grant WM. Further studies on facility of flow through the trabecular meshwork. Arch Ophthalmol 1958;60:523-33.

6 Smith R. A new technique for opening the canal of Schlemm. Br F Ophthalmol 1960;44:370-3.

7 Harms H, Dannheim R. Trabeculotomy-results and problems. Adv Ophthalmol 1970;22:121-31.

8 Dvorak-Theobald G, Kirk HQ. Aqueous pathways in some cases of glaucoma. Am $\mathcal{f}$ Ophthalmol 1956;41:11-21.

9 Krasnov MM. Externalisation of Schlemm's canal (sinusotomy) in glaucoma. Br f Ophthalmol 1968;52:157-61.

0 Gillies WE. Trabeculotomy with fistulisation. A preliminary report of a modified trabeculotomy. Aust F Ophthalmol 1976;4:152-4.

1 Cairns JE. Trabeculectomy. Preliminary report of a new method. Am f Ophthalmol 1968;66:673-9.

2 Khaw PT, Siriwardena D. "New" surgical treatments for glaucoma [Editorial]. Br f Ophthalmol 1999;83:1-2.

13 Krieglstein GK. How new is new, and is it better? [Editorial]. $\mathcal{F}$ Glaucoma 1999;8:279-80.

14 Karlen ME, Sanchez E, Schnyder CC, et al. Deep sclerectomy with collagen implant: medium term results. Br f Ophthalmol 1999;83:6-11. 


\section{Cover illustration: The pinhole camera}

This primitive species of mollusc, the Nautilus is probably over 150 million years old. The invertebrate has changed little over that time and still has a primitive eye, illustrating its successful visual adaptation to its environment. The eye is located behind the tentacles and a pinhole opening is its only external sign. Curiously, there is no lens, no cornea and, in fact, no dioptric element whatsoever. Furthermore, there is no obstruction to the incursion by seawater, thus allowing for a continual exchange with the external pelagic environment. There may be a mucous or gelatinous substance filling the cavity of the eye in life, although its composition is not known nor its presence even confirmed. It probably resembles a vitreous body, but surely must contain an admixture of seawater because of the open fistula.

The eyecup is approximately round with a pupil which has some contractile features to it and with a known pupillary diameter range of at least 0.7-2.25 $\mathrm{mm}$. The weakly contractile pinhole leads into a surprisingly large cavity lined nearly $360^{\circ}$ with cells, individually called retinula, each of which includes a rhabdom at its distal end. These cells are primitive photoreceptors. Other cells in the retina are supporting cells that are directly interspersed between the retinulae, sending processes distally that extend between the rhabdoms. Both types of cells contain pigment granules that can migrate within the cell and are probably used to control light stimulus to the retinula. The axons lead away from the proximal base of the retinula directly to the optic nerve without synapse. Anteriorly, near the pupil, there are pigmented cells without rhabdoms and mucous cells that probably secrete the mucoid-like contents of the chamber. Morphologically resembling an arrogant pugilist, the retinulae lead with their chins by extending the rhabdomal portion of the cell towards the inner cavity, allowing direct seawater contact with the photoreceptive element. In an evolutionary sense, this is a photoreceptive eyespot that has invaginated and developed supporting elements.

Such an eye is not subject to chromatic or spherical aberration, as there is no lens or cornea, but is subject to diffraction limitations of a small opening. Additionally, adequate sensitivity and resolution cannot be obtained simultaneously. As the pupil size becomes smaller the resolution improves up to the point of pupillary diffraction, but the sensitivity decreases dramatically. Not surprisingly, investigators believe the Nautilus actually prefers the light (sensitivity, as they seem to go towards the brighter of two light stimuli in experimental work) confirming that the visual system retains adequate sensitivity even at the expense of resolution.
The Nautilus lives in the Pacific ocean at depths of 150-600 metres along steep underwater cliffs where bioluminescence is a primary source of light. Maximum light transmission at that depth peaks at about $475 \mathrm{~nm}$ corresponding to the absorption spectrum of its visual pigment. Spectral sensitivity, as determined by the absorption spectrum of the extractable visual pigment found in the retina, has been determined to be maximal at about $470 \mathrm{~nm}$.

Although Nautilus shows diurnal activity, they are most active as crepuscular or nocturnal creatures with circadian vertical migrations. These animals are benthic in habit and, given the dark sloping substrate on which they live, they may not know which direction is up. The animals are blessed with statocysts providing information on the direction of gravity as well as the depth, since they would probably implode due to the pressure of seawater when at depths over 800 metres. Strong currents characterise their environment and probably cause these animals to drift long distances, requiring vertical localisation skills.

Bioluminescence is important at these depths, and the fish and other animals found here can be considered point sources. Decaying food matter is the principal diet of the Nautilus and is often associated with bioluminescence. Other animals such as species of shrimp are also attracted to the decaying food matter and many of these shrimp also have bioluminescent characteristics that will attract the Nautilus to its food sources. Nautilus feed slowly and distance vision beyond a few metres is probably of little interest to the animal; they probably do not rely upon vision for protection either since they are encased in a hard outer shell.

Their primitive eye probably has not changed during the 150 million years they have been on earth and this helps us understand how visual systems have evolved. The vertebrates that inhabit the same environment have eyes that are at least superficially similar since both are "camera"-type eyes in contrast with the compound eyes of many other invertebrates, including insects and crustaceans. The "camera"-type eye is also seen in other cephalopods such as the octopus and squid, which are related to the Nautilus. These other cephalopods do have a lens and cornea as the eye has evolved to improve the focus of the image. These cephalopod "camera"type eyes also show convergent evolution since no common ancestor to these invertebrates and vertebrates exist. They have achieved similar results for ocular morphology, but by very different mechanisms, and represent convergent evolution.-IvAN R SCHWAB, UC Davis Department of Ophthalmology, Sacramento, CA, USA (irschwab@ucdavis.edu). 